\title{
Optomechanical Kerker Effect
}

\author{
A. V. Poshakinskiy ${ }^{1, *}$ and A. N. Poddubny ${ }^{1,2}$ \\ ${ }^{1}$ Ioffe Institute, St. Petersburg 194021, Russia \\ ${ }^{2}$ Nonlinear Physics Centre, Research School of Physics and Engineering, Australian National University, \\ Canberra, Australian Capital Territory 2601, Australia
}

(Received 6 August 2018; revised manuscript received 15 November 2018; published 15 January 2019)

Tunable directional scattering is of paramount importance for operation of antennas, routing of light, and design of topologically protected optical states. For visible light scattered on a nanoparticle, the directionality could be provided by the Kerker effect, exploiting the interference of electric and magnetic dipole emission patterns. However, magnetic optical resonances in small sub-100-nm particles are relativistically weak. Here, we predict inelastic scattering with the unexpectedly strong tunable directivity up to 5.25 driven by a trembling of a small particle without any magnetic resonance. The proposed optomechanical Kerker effect originates from the vibration-induced multipole conversion. We also put forward an optomechanical spin-Hall effect, the inelastic polarization-dependent directional scattering. Our results uncover an intrinsically multipolar nature of the interaction between light and mechanical motion and apply to a variety of systems from cold atoms to two-dimensional materials to superconducting qubits. An application for engineering of chiral optomechanical coupling and nonreciprocal transmission at nanoscale is proposed.

DOI: 10.1103/PhysRevX.9.011008

Subject Areas: Metamaterials, Optics, Photonics

\section{INTRODUCTION}

Scattering of light manifests itself in everyday life, fundamental science, and device applications [1]. Elastic Rayleigh scattering governs the blue color of the sky and sea. Inelastic Raman scattering is a workhorse of sensors. The ability to control the direction, frequency, and polarization of the scattered light is essential for optical devices. However, both Rayleigh and Raman scattering usually have a symmetric emission pattern: the waves are symmetrically scattered in two opposite directions, in particular, forward and backward $[2,3]$. The asymmetry can be induced if the particle that scatters light moves. Then, the Doppler effect leads to a difference between the incident and scattered light frequencies [4], which depends on the scattering angle in a highly asymmetric way. As first noted by Raman himself [5], it vanishes for the forward-scattered wave and reaches maximum for the backscattered one. The scattering cross section depends on the angle between the incident light propagation direction and particle velocity, enabling cooling of atomic gases in optical molasses [6]. Still, the asymmetry of the emission intensity pattern remains small

\footnotetext{
*poshakinskiy@mail.ioffe.ru
}

Published by the American Physical Society under the terms of the Creative Commons Attribution 4.0 International license. Further distribution of this work must maintain attribution to the author(s) and the published article's title, journal citation, and DOI. unless the particle velocity becomes comparable to that of light, which is realized, e.g., for Compton scattering of $\mathrm{x}$ rays [7].

A simpler mechanism to achieve strong scattering directionality was proposed by Kerker et al. [8]. Rather than using mechanical motion, it requires a scatterer to possess both electric dipole (ED) and magnetic dipole (MD) susceptibilities. Since the electric field of these two modes is of the opposite spatial parity, their interference enables directional forward or backward scattering depending on the relative phase [9-11]. Thus, implementation of the Kerker effect requires magnetic response of the same strength as the electric one. At optical frequencies this becomes challenging since magnetic dipole transitions are intrinsically relativistically weak [12]. A promising recently emerged workaround is provided by submicron-size high-refractive-index dielectric nanoparticles [13-15] hosting both magnetic and electric Mie resonances. For instance, Huygens metasurfaces of Si nanodisks that transmit light forward changing its phase without reflection open new avenues for wave front control at the nanoscale $[16,17]$. However, optical Kerker effect for the particles smaller than the wavelength in the medium $\sim 100 \mathrm{~nm}$ is still unfeasible.

Here, we uncover a deep nexus of the motion-induced scattering directionality and the Kerker effect. We put forward an optomechanical Kerker effect, where strong tunable directionality is achieved for light scattered by a small particle without any magnetic response that trembles in space. Our main concept is sketched in Fig. 1. The incident 
(a)

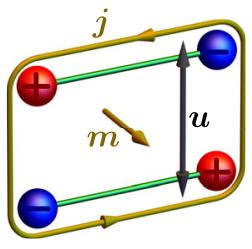

(b) $\boldsymbol{E}^{\prime} e^{-i(\omega+\Omega) t}$

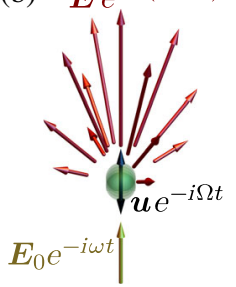

(c)

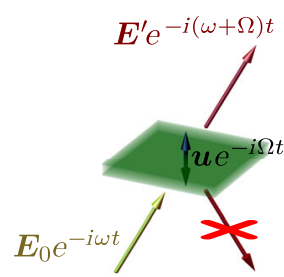

FIG. 1. (a) A sketch of the motion-induced multipole conversion. Trembling of an oscillating dipole in the space $\boldsymbol{u}$ leads to appearance of an electric quadrupole and a current curl. The latter yields a magnetic dipole $\boldsymbol{m}$. (b) A sketch directional inelastic light scattering on a trembling particle. (c) A sketch of the trembling resonant layer, an optomechanical analogue of Huygens surface.

wave excites electric dipole polarization, that oscillates in time. Trembling of the electric dipole in the direction transverse to its polarization induces the loop electric current $\boldsymbol{j}$ with nonzero magnetic momentum $\boldsymbol{m}$ as well as the electric quadrupole (EQ) momentum. Interference of $\mathrm{ED}$ and $\mathrm{MD}+\mathrm{EQ}$ contributions results in unidirectional scattering as shown in Figs. 1(b) and 1(c). While the idea to use motion-induced conversion of electric dipole to magnetic dipole seems straightforward, a naive expectation would be that the magnetic dipole is relativistically weaker than the electric one and their interference cannot result in any significant directionality. We found that magnetic and electric dipole components counterintuitively are of the same order when inelastic light scattering is considered. To demonstrate this, we have developed a novel theoretical framework of multipolar resonant optomechanics. It incorporates the effect of the resonant dispersion of the moving medium on the multipolar emission in a rigorous nonperturbative fashion and goes beyond previous approaches [18-20] restricted to nonresonant scatterers. Our predictions are quite general and apply both for particles and for thin layers, as shown in Figs. 1(b) and 1(c). We also put forward an optomechanical spin-Hall effect, i.e., directional inelastic scattering of light depending on its circular polarization. An application of the uncovered effects for design of chiral optomechanical coupling at nanoscale and nonreciprocal transmission is proposed.

\section{DIRECTIONAL INELASTIC SCATTERING}

\section{A. Nonresonant inelastic backscattering}

We start with the qualitative geometrical consideration to reveal a drastic difference in the angular patterns of elastic and inelastic scattering. Figure 2(a) sketches the plane wave with the frequency $\omega$ that is scattered on a small particle trembling at the frequency $\Omega$ along the incident light propagation direction. The incident light induces dipole polarization of the particle that then emits light in a different direction. The shift of the particle in the real space $u_{z}(t)$ gives rise to an additional time-dependent phase

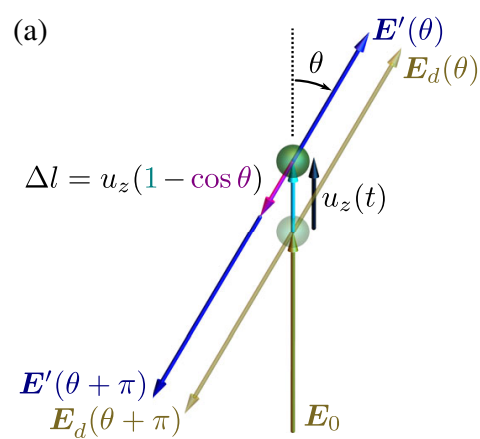

(b)

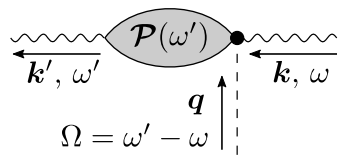

(c)

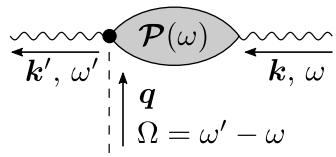

FIG. 2. (a) A sketch of light scattering on a trembling particle. The incident and elastically scattered light are shown by yellow color, inelastically scattered light is shown by blue color. Inelastic scattering is caused by the temporal modulation of the optical path (cyan and magenta arrows) due to particle displacement. (b), (c) Diagrammatic representation for the inelastic light scattering on a trembling particle. Wavy lines denote photons, bubbles correspond to the dressed polarization operator of the particle at rest, dashed lines represent mechanical displacement, solid dot stands for the optomechanical interaction given by Eq. (A5).

of the scattered light $\phi(\theta, t)=(1-\cos \theta)(\omega / c) u_{z}(t)$, where the two terms are illustrated by cyan and magenta arrows in Fig. 2(a) and $\theta$ is the scattering angle. The electric field of the scattered wave reads $\boldsymbol{E}^{\prime}(\theta)=\boldsymbol{E}_{d}(\theta) e^{i \phi(\theta, t)}$, with $\boldsymbol{E}_{d}(\theta)$ being the electric field of the light scattered by the particle at rest. Taking the particle displacement in the form $u_{z}(t)=u_{z} e^{-i \Omega t}+$ c.c. and expanding the scattered field into series over $u_{z}$, one obtains harmonics at the frequencies $\omega+p \Omega$ with integer $p$. We suppose that the vibration amplitude is small. Then, the electric field of the harmonic at the initial light frequency $\omega$, that describes the elastic light scattering, coincides with $\boldsymbol{E}_{d}(\theta)$. Its angular dependence is governed by the well-known electric dipole radiation pattern that yields equal amplitudes of forward and backward scattering [21]. The linear-in- $u_{z}$ terms yield the harmonics at anti-Stokes- and Stokes-shifted frequencies $\omega \pm \Omega$ that describe inelastic scattering. Their intensities read

$$
I^{\prime}(\theta) \approx(\omega / c)^{2}\left|u_{z}\right|^{2}(1-\cos \theta)^{2} I_{d}(\theta)
$$

where $I_{d}(\theta) \propto\left|E_{d}(\theta)\right|^{2}$ is the intensity of elastic dipole scattering. In stark contrast to elastic scattering, the inelastic scattering is strongly anisotropic. In forward scattering geometry, the particle shift does not change the optical path. Thus, Stokes and anti-Stokes light intensities vanish for $\theta=0$. The inelastic scattering is the most intensive in the backscattering geometry, $\theta=\pi$, when the optical path change is maximal.

\section{B. Multipolar resonant inelastic scattering}

The above geometric consideration predicting the inelastic scattering asymmetry has a crucial limitation: 
It is applicable only to the particle with the frequencyindependent polarizability. Indeed, in the case of resonant optical response, the elastic scattering intensity $I_{d}$ strongly depends on the light frequency. Yet, it is completely unclear which frequency to choose in Eq. (1): that of the incident light $\omega$ or that of the scattered light $\omega^{\prime}=\omega \pm \Omega$.

To resolve this fundamental problem, we develop a rigorous theory of light interaction with a polarization of a trembling medium. We stress that the inelastic scattering considered here is caused by the motion-induced modulation of the interaction between light and scatterer, in contrast to conventional resonant Raman scattering that is due to modulation of the eigenenergies of the scatterer itself. We derive the general expression for inelastic scattering intensity in Appendix B while here we focus on the case of a small trembling object described by the frequencydependent electric dipole polarizability tensor $\boldsymbol{\alpha}(\omega)$. In that case, the amplitude of inelastic scattering comprises two terms that are diagrammatically represented in Figs. 2(b) and 2(c). They show the vibration quantum (dashed line) being absorbed or emitted either before or after the medium polarization (bubble) is induced. This reflects the change of the optical path before and after the scattering on the particle; see cyan and magenta arrows in Fig. 2(a), respectively. Concomitantly, the two terms in the inelastic scattering amplitude feature polarization operators $\mathcal{P}$ at the frequency of scattered light $\omega^{\prime}$ and at that of the incident light $\omega$. It is the interference of these two contributions, which can be both constructive and destructive for objects with resonant permittivity, that leads to the strong directivity of the scattered light.

For the electric field at the anti-Stokes-shifted frequency $\omega^{\prime}=\omega+\Omega$ at $r \rightarrow \infty$, we get

$$
\begin{aligned}
\boldsymbol{E}^{\prime}(\boldsymbol{r})= & \frac{i \omega^{\prime 2} e^{i \omega^{\prime} r / c}}{c^{3} r}\left[\omega^{\prime}\left(\boldsymbol{n}_{0} \cdot \boldsymbol{u}\right) \boldsymbol{\alpha}\left(\omega^{\prime}\right) \boldsymbol{E}_{0}-\omega(\boldsymbol{n} \cdot \boldsymbol{u}) \boldsymbol{\alpha}(\omega) \boldsymbol{E}_{0}\right. \\
& \left.-\Omega\left(\boldsymbol{u} \cdot \boldsymbol{E}_{0}\right) \boldsymbol{\alpha}\left(\omega^{\prime}\right) \boldsymbol{n}_{0}-\Omega\left(\boldsymbol{n} \cdot \boldsymbol{\alpha}(\omega) \boldsymbol{E}_{0}\right) \boldsymbol{u}\right]_{\perp}
\end{aligned}
$$

where $\boldsymbol{n}=\boldsymbol{r} / r, \boldsymbol{n}_{0}$ and $\boldsymbol{E}_{0}$ are the propagation direction and electric field of the incident wave, subscript $\perp$ indicates that the perpendicular component with respect to $\boldsymbol{n}$ should be taken, $[\boldsymbol{E}]_{\perp}=-\boldsymbol{n} \times(\boldsymbol{n} \times \boldsymbol{E})$. The field Eq. (2) can be decomposed into electric dipole $\boldsymbol{p}$, quadrupole $\boldsymbol{Q}$, and magnetic dipole $\boldsymbol{m}$ contributions oscillating at the frequency $\omega^{\prime}$ with the amplitudes

$$
\begin{aligned}
\boldsymbol{d} & =\frac{i}{c} \boldsymbol{\alpha}\left(\omega^{\prime}\right)\left[\omega\left(\boldsymbol{n}_{0} \cdot \boldsymbol{u}\right) \boldsymbol{E}_{0}-\Omega \boldsymbol{u} \times\left(\boldsymbol{n}_{0} \times \boldsymbol{E}_{0}\right)\right], \\
\boldsymbol{Q} & =3 \boldsymbol{\alpha}(\omega) \boldsymbol{E}_{0} \otimes \boldsymbol{u}+3 \boldsymbol{u} \otimes \boldsymbol{\alpha}(\omega) \boldsymbol{E}_{0}-2 \boldsymbol{I}\left(\boldsymbol{u} \cdot \boldsymbol{\alpha}(\omega) \boldsymbol{E}_{0}\right), \\
\boldsymbol{m} & =\frac{i}{2 c}(\omega-\Omega)\left[\boldsymbol{\alpha}(\omega) \boldsymbol{E}_{0} \times \boldsymbol{u}\right],
\end{aligned}
$$

where $(\boldsymbol{a} \otimes \boldsymbol{b})_{\alpha \beta}=a_{\alpha} b_{\beta}$ and $\boldsymbol{I}$ is the identity matrix. All the multipole terms are of the same order; however, the induced electric dipole is proportional to the polarizability at the scattered light frequency $\boldsymbol{\alpha}\left(\omega^{\prime}\right)$ while electric quadrupole and magnetic dipole are determined by $\boldsymbol{\alpha}(\omega)$. Therefore, the frequency dependence of polarizability can be exploited to tune $\boldsymbol{d}, \boldsymbol{m}$, and $\boldsymbol{Q}$ to the Kerker condition.

\section{Optomechanical Kerker effect}

Now we analyze in detail the direction pattern Eq. (2) for light scattered on a trembling particle with the isotropic resonant electric dipole polarizability $\alpha(\omega)$. We focus on the anti-Stokes component at the frequency $\omega^{\prime}=\omega+\Omega$. Similar results for the Stokes component are obtained by inverting the sign of $\Omega$ and complex conjugation of the particle displacement vector $\boldsymbol{u}$. First, we neglect the last two terms in the right-hand side of Eq. (2) proportional to the parameter $\Omega / \omega$, that is small for realistic systems. Figure 3 shows the radiation pattern of the light scattered on the particle trembling along the propagation direction of the linearly polarized incident wave. Figure 3(a) shows the usual elastic electric dipole scattering at the frequency $\omega$, while Figs. 3(c) and 3(d) correspond to the inelastic scattering to the frequency $\omega^{\prime}$. Figure 3(c) shows the contribution of the first electric dipole term in Eq. (2) to the scattered field, while Fig. 3(d) corresponds to the second term in Eq. (2) and a combination of magnetic dipole and electric quadrupole radiation. The total scattering intensity is a superposition of the patterns Figs. 3(c) and 3(d) with the coefficients $\alpha\left(\omega^{\prime}\right)$ and $\alpha(\omega)$, respectively. While the frequencies $\omega$ and $\omega^{\prime}$ are close, the corresponding polarizabilities can differ strongly in the vicinity of the material resonance. Figures 3(b) and 3(e) show the two limiting cases when $\alpha\left(\omega^{\prime}\right)= \pm \alpha(\omega)$. In the nonresonant case, $\alpha\left(\omega^{\prime}\right)=\alpha(\omega)$, the interference of electric dipole,

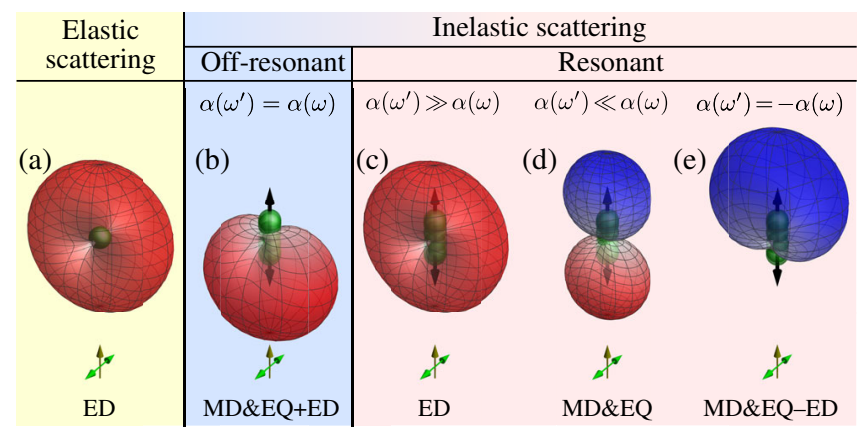

FIG. 3. Radiation pattern for (a) elastic light scattering and (b)-(e) inelastic scattering by a trembling particle for different ratios of the polarizabilities at initial and scattered frequencies $\alpha(\omega)$ and $\alpha\left(\omega^{\prime}\right)$. The interference of the electric dipole (c) and magnetic dipole and electric quadrupole (d) patterns results in directional inelastic forward (e) and backward (b) scattering. The light is incident from the bottom (yellow arrow) and is linearly polarized (green arrow), the particle trembles along the light propagation direction (black arrow). Red and blue colors indicate the sign of electric field. 
magnetic dipole, and electric quadrupole radiation leads to the complete suppression of forward inelastic scattering, Fig. 3(c). In the opposite case of strong frequency dependence of polarizability when $\alpha\left(\omega^{\prime}\right)=-\alpha(\omega)$, Fig. 3(d), the inelastic backscattering vanishes.

Using Eq. (2) we evaluate the cross section of the inelastic scattering for unpolarized light,

$$
\frac{d \sigma}{d o}=\frac{\omega^{6}}{2 c^{6}}\left|\left[\alpha\left(\omega^{\prime}\right) \boldsymbol{n}_{0}-\alpha(\omega) \boldsymbol{n}\right] \cdot \boldsymbol{u}\right|^{2}\left[1+\left(\boldsymbol{n}_{0} \cdot \boldsymbol{n}\right)^{2}\right],
$$

where $d o$ is the solid angle for scattered light direction. In the nonresonant case when $\alpha\left(\omega^{\prime}\right)=\alpha(\omega)$ and $\boldsymbol{n}_{0} \| \boldsymbol{u}$, we recover the geometric optics result Eq. (1) with $d \sigma \propto$ $(1-\cos \theta)^{2}$ and suppressed forward scattering.

The asymmetry of the light scattering pattern can be quantified by the directivity $D(\boldsymbol{n})=4 \pi\left(\int d \sigma\right)^{-1} d \sigma / d o$ [22] In the considered geometry, $\boldsymbol{u} \| \boldsymbol{n}_{0}$, the directivity for forward $\left(\boldsymbol{n}=\boldsymbol{n}_{0}\right)$ and backward $\left(\boldsymbol{n}=-\boldsymbol{n}_{0}\right)$ scattering reads

$$
D\left( \pm \boldsymbol{n}_{0}\right)=\frac{15\left|\alpha\left(\omega^{\prime}\right) \mp \alpha(\omega)\right|^{2}}{10\left|\alpha\left(\omega^{\prime}\right)\right|^{2}+4|\alpha(\omega)|^{2}} .
$$

For the nonresonant case, when $\alpha\left(\omega^{\prime}\right)=\alpha(\omega)$, the forward scattering is absent while the backward directivity reaches $30 / 7$. The maximal value of forward (backward) directivity is 5.25, which is achieved when $\alpha\left(\omega^{\prime}\right)= \pm(2 / 5) \alpha(\omega)$. Thus, the directivity of the optomechanical Kerker effect surpasses the limiting value of 3 for the classical Kerker effect, because the electric quadrupole contribution is additionally involved [11]. Even larger values of directivity $[23,24]$ can be achieved in second- and higher-order inelastic scattering processes, where the scattered light contains higher electric and magnetic multipoles.

For numerical demonstration, we consider the simplistic general model of the particle characterized by the resonant polarizability

$$
\alpha(\omega)=\frac{A}{\omega-\omega_{x}+i \Gamma},
$$
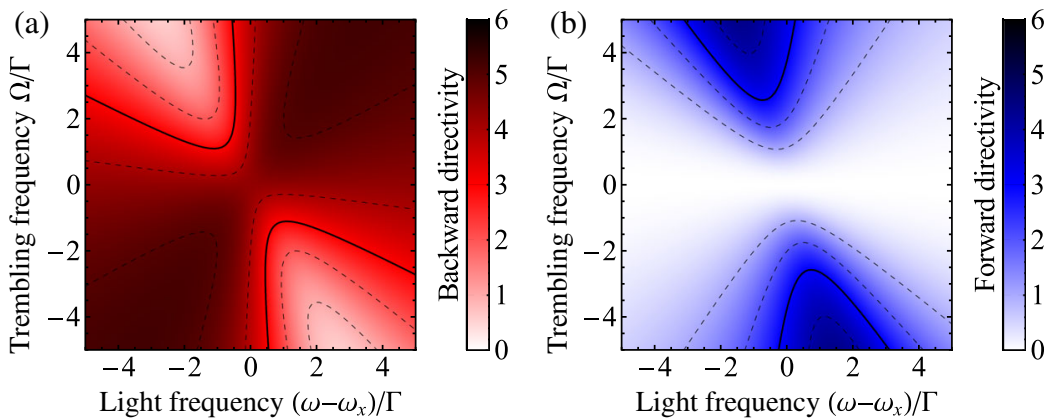

where $A$ is a constant, $\omega_{x}$ is the resonance frequency, and $\Gamma$ is the resonance width. Such dependence corresponds to, e.g., exciton resonance in quantum dots and transitional metal dichalcogenide monolayers, electron transitions in cold atomic gases, plasmon resonance in graphene, resonances in atomic nuclei probed by Mössbauer $\gamma$-ray spectroscopy, and superconducting resonators for radiofrequency electromagnetic field; see Sec. VI. Figures 4(a) and 4(b) show the color plots of the directivity of backward and forward anti-Stokes $(\Omega>0)$ and Stokes $(\Omega<0)$ light scattering depending on the incident light frequency $\omega$ and the trembling frequency $\Omega$. In the dark areas bounded by the solid lines, the directivity is larger than 3 , which can be termed a super-Kerker effect. For backward and forward scattering it is realized when initial and scattered light frequencies $\omega$ and $\omega^{\prime}=\omega+\Omega$ are located on the same side or on the opposite sides of the resonance, respectively. Shown in Fig. 4(c) is the degree of directivity $\left[D\left(\boldsymbol{n}_{0}\right)-D\left(-\boldsymbol{n}_{0}\right)\right] /\left[D\left(\boldsymbol{n}_{0}\right)+D\left(-\boldsymbol{n}_{0}\right)\right]$. Red and blue colors indicate predominance of the backward and forward scattering, respectively. The degree of directivity reaches \pm 1 if $\alpha\left(\omega^{\prime}\right)=\mp \alpha(\omega)$, which is realized at $\Omega=2\left(\omega_{x}-\omega\right) \gg \Gamma$ and $\Omega \rightarrow 0$, respectively.

\section{OPTOMECHANICAL SPIN HALL EFFECT}

The interference of the electric and magnetic modes is known to give rise to a strong circular polarization of the scattered light upon excitation with a linearly polarized light $[25,26]$. Conversely, photons with opposite circular polarizations scatter in different directions. This is termed an optical spin-Hall effect $[27,28]$ in analogy with the spindependent scattering of electrons in solids [29]. Here, we put forward an optomechanical spin-Hall effect, i.e., inelastic polarization-dependent directional scattering on a trembling particle.

The two first terms in Eq. (2) are dominant and yield the scattered light with the same polarization as the incident. Optomechanical spin-Hall effect results from the last two terms, which give a small correction of the order $\Omega / \omega$ describing linear-to-circular polarization conversion. The

FIG. 4. (a) Backward and (b) forward directivity of nonpolarized light inelastically scattered by a trembling resonant particle depending on the incident light frequency $\omega$ and trembling frequency $\Omega$. Solid line shows the directivity equal to 3 that limits usual Kerker effect. (c) Degree of directivity $\left[D\left(\boldsymbol{n}_{0}\right)-D\left(-\boldsymbol{n}_{0}\right)\right] /\left[D\left(\boldsymbol{n}_{0}\right)+D\left(-\boldsymbol{n}_{0}\right)\right]$. 
circular polarization degree of the plane wave with the electric field amplitude $\boldsymbol{E}$ can be defined as $P_{c}(\boldsymbol{n})=$ in $\cdot\left[\boldsymbol{E} \times \boldsymbol{E}^{*}\right] /|\boldsymbol{E}|^{2}$. Substituting here the scattered wave from Eq. (2) we obtain for the case of nonpolarized incident light

$$
P_{c}=\frac{\Omega}{\omega} \frac{2 \boldsymbol{n} \times \boldsymbol{n}_{0}}{1+\left(\boldsymbol{n}_{0} \cdot \boldsymbol{n}\right)^{2}} \operatorname{Im} \frac{\left[\alpha\left(\omega^{\prime}\right)+\left(\boldsymbol{n}_{0} \cdot \boldsymbol{n}\right) \alpha(\omega)\right] \boldsymbol{u}}{\left[\alpha\left(\omega^{\prime}\right) \boldsymbol{n}_{0}-\alpha(\omega) \boldsymbol{n}\right] \cdot \boldsymbol{u}},
$$

where we keep linear in $\Omega / \omega$ terms only. Equation (7) indicates two possible origins of circular polarization: (i) the phase difference of the polarizabilities $\alpha(\omega)$ and $\alpha\left(\omega^{\prime}\right)$ and (ii) the phase difference of the components of the displacement vector $\boldsymbol{u}$. The first mechanism is likely to contribute in the vicinity of the material resonance where the phase of $\alpha$ rapidly changes by $\pi$. The second mechanism is realized even away from the resonances; however, it requires the particle trembling around a circle or an ellipse rather than just along one axis.

Figure 5 shows by red and blue color the circular polarization degree of the anti-Stokes-scattered light for different particle trembling directions, indicated on the left, and different relations between polarizabilities at the frequencies of incident and scattered light, indicated on the top. The plots on the gray background show the cases where both mechanisms (i) and (ii) are absent, so the circular polarization does not emerge. The first mechanism is realized for the plots on the blue background, where we as an example assume $\alpha\left(\omega^{\prime}\right)=i \alpha(\omega)$. For $\boldsymbol{u} \| \boldsymbol{n}_{0}$, see Fig. 5(d), the dependence of $P_{c}$ on the azimuthal angle is described by the second angular harmonic, so $P_{c}$ is inverted when the incident light with the perpendicular polarization is considered. For unpolarized excitation, the circular polarization vanishes in agreement with Eq. (7). Figure 5(h) shows the angular pattern of $P_{c}$ for light scattered by the particle trembling perpendicularly to the direction of incident light and $\boldsymbol{E}_{0} \| \boldsymbol{u}$. For the other linear polarization of the incident light (not shown), the conversion to circular polarization is absent. Therefore, even for the nonpolarized incident light the circular polarization of scattered light persists and it is described by Eq. (7).

Now we turn to the second mechanism of the generation of circular polarization, that is realized for the plots on the yellow background. Figures 5(i)-5(k) illustrate the circular polarization of the light scattered by the particle trembling around a circular trajectory in the plane perpendicular to the incident light direction. Then, the forward- and backwardscattered light reveal opposite signs of circular polarization, except for the case of Fig. 5(i) when the scattered wave is of the order of the small parameter $\Omega / \omega$ and linearly polarized; see Eq. (2). Figures 5(m)-5(o) and 5(q)-5(s) show the pattern of the circular polarization degree of the light scattered by the particle trembling around a circular trajectory in the plane parallel to the incident light $\alpha\left(\omega^{\prime}\right) \gg \alpha(\omega) \quad \alpha\left(\omega^{\prime}\right) \ll \alpha(\omega) \quad \alpha\left(\omega^{\prime}\right)=\alpha(\omega) \quad \alpha\left(\omega^{\prime}\right)=\mathrm{i} \alpha(\omega)$

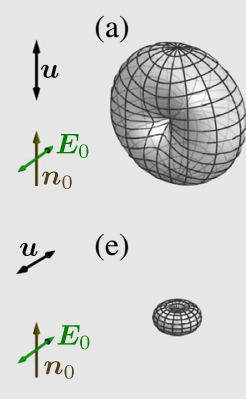

(b)

(c)
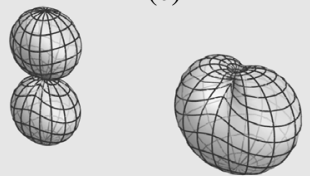

(d)

(f)

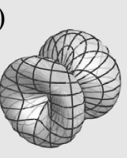

(g)

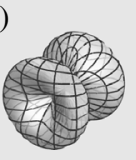

(h)
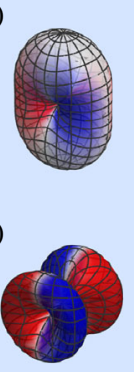

(i)

(j)

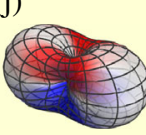

(k)

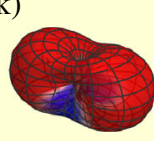

(1)

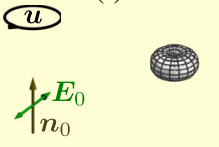

(m)

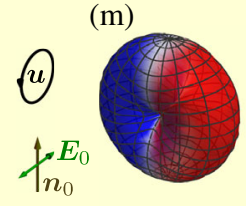

(n)

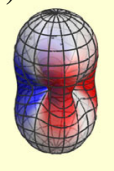

(o)

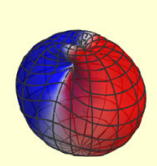

(p)
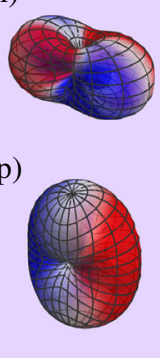

(q)

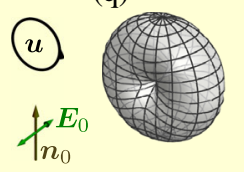

(r)

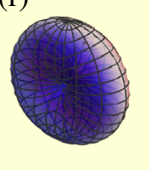

(s)

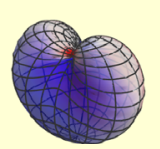

(t)

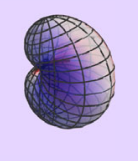

Circular polarization degree $[\Omega / \omega]$

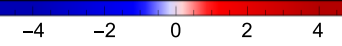

FIG. 5. The anti-Stokes light scattering pattern with the degree of circular polarization marked by red and blue colors. Light is incident from the bottom and linearly polarized, see yellow and green arrows on the left, respectively. Rows correspond to different trembling directions indicated by black arrows on the left. Columns correspond to different relations between particle polarizabilities at the frequencies of incident and scattered light, $\alpha(\omega)$ and $\alpha\left(\omega^{\prime}\right)$, indicated in the top. The colored areas represent different origins of the optomechanical spin-Hall effect: phase difference between the polarizabilities $\alpha(\omega)$ and $\alpha\left(\omega^{\prime}\right)$ (blue color), the phase difference between components of the displacement vector $\boldsymbol{u}$ (yellow color), or their combined action (purple color).

propagation direction. The circular polarization sign depends on whether the light is scattered to the left or to the right with respect to the plane of trembling. Finally, if both optomechanical spin-Hall effect mechanisms are present, see plots on the purple background, their interplay leads to a strong asymmetry of both the scattering intensity pattern and the circular polarization pattern.

\section{OPTOMECHANICAL HUYGENS SURFACES}

A two-dimensional planar array of scatterers tuned to the Kerker condition is known to demonstrate no forward or backward scattering $[16,17,30]$. When the backscattering is suppressed it realizes a Huygens' surface that transmits 
light without reflection and modifies only its phase. An optomechanical analog of the Huygens' surface is a thin layer with a resonant dipole polarizability that trembles along its normal; see Fig. 6(a).

Since the flexural layer vibrations can possess an inplane wave vector $\boldsymbol{q}$, the direction of scattered light can differ from that of the incident light. It is determined from the conservation of the in-plane wave vector component in the process of anti-Stokes (Stokes) scattering, $\boldsymbol{k}^{\prime}=\boldsymbol{k} \pm \boldsymbol{q}$, where $k=(\omega / c) \cos \theta$ and $k^{\prime}=\left(\omega^{\prime} / c\right) \cos \theta^{\prime}$ are the inplane wave vectors of the incident and scattered light, and $\theta$ and $\theta^{\prime}$ are the angles between the light propagation direction and the layer normal. The electric field amplitudes of the anti-Stokes forward- $(\rightarrow)$ and backward- $(\hookleftarrow)$ scattered light are given by the diagrams of Figs. 2(b) and 2(c) and read $E_{\sigma}^{\prime \rightarrow(\hookleftarrow)}=\sum_{\sigma^{\prime}} S_{\sigma \sigma^{\prime}}^{\rightarrow(\hookleftarrow)} E_{0, \sigma^{\prime}} u_{\boldsymbol{q}, z}$, where $\sigma, \sigma^{\prime}$ enumerates two polarizations, $s$ and $p$. The Jones matrix $S_{\sigma \sigma^{\prime}}^{\rightarrow(\leftrightarrow)}$ with the elements (see Appendix C for calculation details)

$S_{s s}^{\rightarrow(\hookleftarrow)}=i \frac{\omega^{\prime}}{c} \cos \theta \cos \phi\left[r_{s}\left(\theta^{\prime}, \omega^{\prime}\right) \mp r_{s}(\theta, \omega)\right]$,

$S_{p s}^{\rightarrow(\hookleftarrow)}=i \frac{\omega^{\prime}}{c} \frac{\cos \theta \sin \phi}{\cos \theta^{\prime}}\left[r_{p}\left(\theta^{\prime}, \omega^{\prime}\right) \mp r_{s}(\theta, \omega)\right]$,

$S_{s p}^{\rightarrow(\leftrightarrow)}=-i \frac{\omega^{\prime}}{c} \sin \phi\left[r_{s}\left(\theta^{\prime}, \omega^{\prime}\right) \mp r_{p}(\theta, \omega)\right]$,

$S_{p p}^{\rightarrow(\leftrightarrow)}=i \frac{\omega^{\prime}}{c} \frac{\cos \phi-\sin \theta \sin \theta^{\prime}}{\cos \theta^{\prime}}\left[r_{p}\left(\theta^{\prime}, \omega^{\prime}\right) \mp r_{p}(\theta, \omega)\right]$

describes polarization conversion, where $r_{s}(\theta, \omega)$ and $r_{p}(\theta, \omega)$ are the reflection coefficients for oblique incidence of $s$ - and $p$-polarized light on the layer at rest, and $\phi$ is the angle between the in-plane wave vectors $\boldsymbol{k}$ and $\boldsymbol{k}^{\prime}$. Similarly to the optomechanical Kerker effect for the trembling particle, the forward (backward) scattering on a trembling layer vanishes when $r_{s(p)}\left(\theta^{\prime}, \omega^{\prime}\right)= \pm r_{s(p)}(\theta, \omega)$. The power of the anti-Stokes light scattered forward (backward) into the solid angle do by the unit area of the layer for the case of unpolarized excitation with the intensity $I_{0}$ reads

$$
\frac{d I^{\prime}}{d o}=\frac{\omega^{\prime 2}}{c^{2}} \frac{\cos ^{2} \theta^{\prime}}{\cos \theta} \frac{1}{2} \sum_{\sigma \sigma^{\prime}}\left|S_{\sigma \sigma^{\prime}}^{\rightarrow(\hookleftarrow)}\right|^{2}\left|u_{q}\right|^{2} I_{0} .
$$

Figures 6(b) and 6(c) show the forward- and backwardscattered power for the case of normal incidence, $\theta=0$, and constant $\left|u_{q}\right|^{2}$. Both plots feature two resonances indicated by dashed lines: the resonance for incident light at $\omega=\omega_{x}$ and the resonance for scattered light at $\omega+\Omega=\omega_{x}$. Since the vibration frequency $\Omega$ increases with the vibration wave vector $q=\left(\omega^{\prime} / c\right) \sin \theta^{\prime}$, the two resonances split with an increase of the scattering angle $\theta^{\prime}$. The main result of Figs. 6(b) and 6(c) is that the forward scattering involving vibration with $\boldsymbol{q}=0$, i.e., for $\theta^{\prime}=0$, is suppressed while the backward scattering at $\theta^{\prime}=0$ is increased. The absence of the forward-scattered wave in the limit $\Omega, \boldsymbol{q} \rightarrow 0$ has a clear physical interpretation: in the quasistatic picture, the shift of the layer as a whole affects the reflected plane wave but does not affect the transmitted one.

\section{RESONANT CHIRAL OPTOMECHANICS}

We now discuss how the tunable directional scattering demonstrated above can be used for the design of chiral optomechanical interaction at the nanoscale. Chiral quantum optics has recently emerged as a concept to couple the wave vector of propagating light to the spin of quantum emitters [32,33], enabling nonreciprocal transmission of light and one-way superradiance. The chiral optomechanical coupling, i.e., when the light propagating in opposite directions interacts with mechanical modes differently, can be induced by an optical pump with a propagating (a)

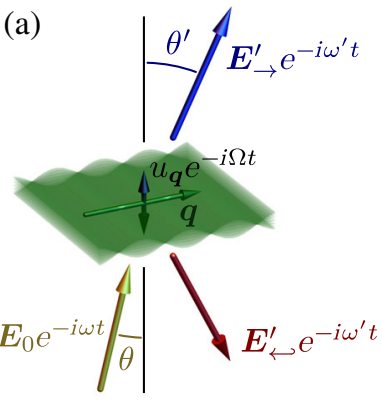

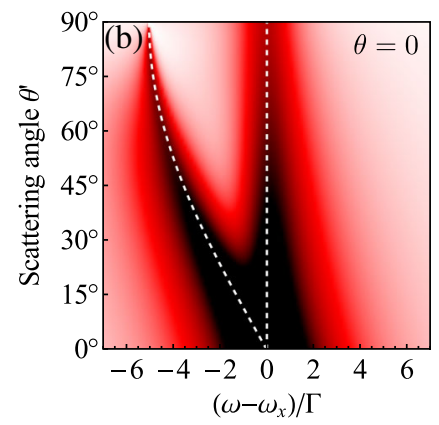
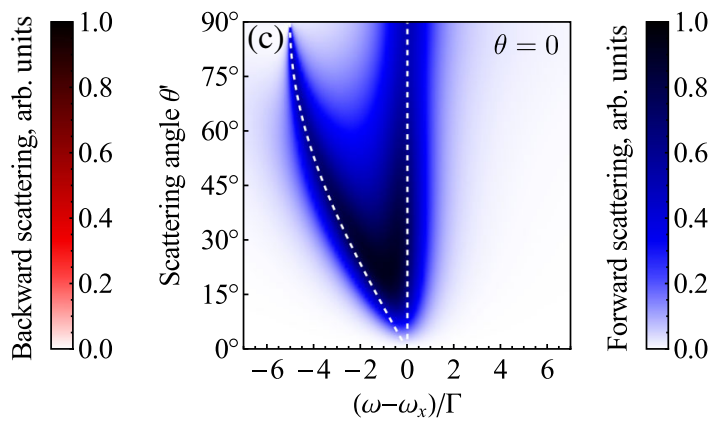

FIG. 6. (a) A sketch of light scattering on a resonant layer with flexural vibrations. Color maps of (b) backward- and (c) forwardscattered power for the case of normal incidence as a function of incident light frequency and scattering angle $\theta^{\prime}$. Dashed lines indicate the resonances for incident and scattered light. The reflection coefficient of resonant layer was taken in the form $r_{s(p)}(\omega, \theta)=$ $-i \Gamma_{s(p)} /\left(\omega-\omega_{x}+i \Gamma_{s(p)}\right)$, with $\Gamma_{s}=\Gamma / \cos \theta$ and $\Gamma_{p}=\Gamma \cos \theta$ [31]. The linear dispersion of phonons was assumed, $\Omega=s|\boldsymbol{q}|$, with $(s / c)\left(\omega_{x} / \Gamma\right)=5$. 
wave $[34,35]$. The pump wave vector breaks the parity and time-inversion symmetries and imposes different phasematching conditions for copropagating and counterpropagating waves, enabling nonreciprocity in optomechanical waveguides and circular resonators [36-38]. However, this mechanism cannot be used in subwavelength systems where the phase-matching condition is relaxed.

In the optomechanical Kerker effect demonstrated above for subwavelength particles, the light scattering amplitude Eq. (2) depends on the propagation direction of both incident and scattered waves. This results in dominance of the backward or forward scattering indicating that the particle vibrations are preferentially coupled to backward or forward propagating light and enabling chiral optomechanical effects even for strongly subwavelength systems.

To illustrate applications of such intrinsically chiral coupling, we consider the simplest optomechanical system depicted in Fig. 7(a). It consists of a one-dimensional waveguide and a small resonant particle located inside or in its vicinity and interacting with the guided mode. The particle is confined in such a way that it can move along the waveguide with the eigenfrequency $\Omega_{r}$ and some mechanical damping $\gamma$. In the absence of particle motion, the light propagation through the waveguide can be characterized by the reflection coefficient $r(\omega)$ and transmission coefficient $t(\omega)=1+r(\omega)$. The system is pumped from the bottom by the laser light with frequency $\omega_{0}$ and electric field amplitude $E_{0}$. We study pump-dependent reflection and transmission coefficients for additional probe optical waves at the frequency $\omega$, incident either from the top or from the bottom.

Probe reflection and transmission are modified due to the processes where in the presence of pump field the probe photon is converted to a mechanical vibration quantum, which is then converted back to a photon. The amplitude of the latter process is, as it was calculated in the previous sections, proportional to $r(\omega)-s^{\prime} r\left(\omega_{0}\right)$, where $s^{\prime}= \pm 1$

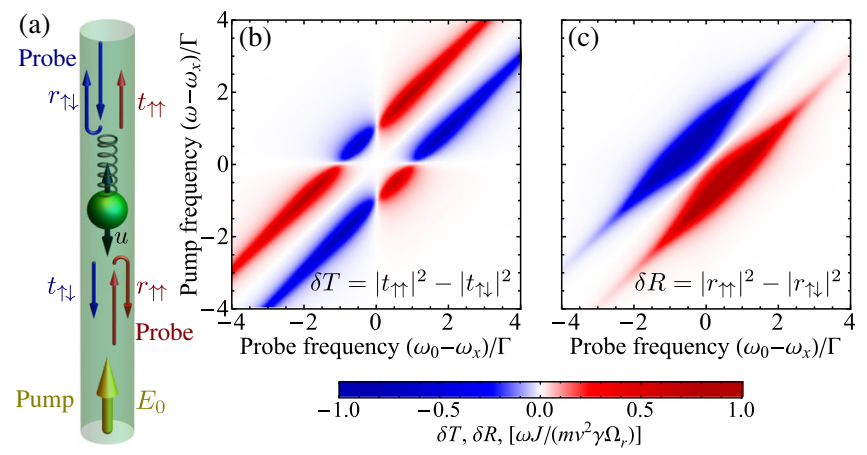

FIG. 7. (a) A sketch of an optomechanical system consisting of a waveguide and a small particle that can oscillate in space along its axis. Color plots of the optomechanically induced probe light (b) nonreciprocal transmission and (c) asymmetrical reflection as a function of pump and probe light frequencies. Calculation is made for a resonant particle with the reflection coefficient $r(\omega)=-i \Gamma /\left(\omega-\omega_{x}+i \Gamma\right), \Omega_{r}=\Gamma$, and $\gamma=0.2 \Gamma$. corresponds to the scattered photon propagating in the same (opposite) direction as the pump light. The amplitude of the reciprocal process of light-vibration conversion is calculated similarly. It consists of two contributions corresponding to processes shown in Figs. 2(b) and 2(c) and is proportional to $r(\omega)+s r^{*}\left(\omega_{0}\right)$, where $s= \pm 1$ corresponds to the incident probe photon propagating in the same (opposite) direction as the pump light. It is instructive to consider a nonresonant case when $r$ is frequencyindependent and purely imaginary. Then, the conversion between the probe light and the vibration occurs only at $s$, $s^{\prime}=-1$; i.e., only the probe wave which propagates in the direction opposite to that of the pump can couple to the mechanical mode.

The optomechanical correction to the light transmission $\left(s^{\prime}=s\right)$ and reflection $\left(s^{\prime}=-s\right)$ coefficients reads

$$
\delta S_{s^{\prime} s}=\frac{i \omega J}{m v^{2}} \frac{\left[r(\omega)-s^{\prime} r\left(\omega_{0}\right)\right]\left[r(\omega)+s r^{*}\left(\omega_{0}\right)\right]}{\left(\omega-\omega_{0}+i \gamma\right)^{2}-\Omega_{r}^{2}},
$$

where $J=\left|E_{0}\right|^{2} /(2 \pi)$ is the power of the pump light, $m$ is the particle mass, and $v$ is the speed of the waveguide mode. Equation (10) yields $\delta S_{++} \neq \delta S_{--}$, meaning that the light transport is nonreciprocal. The transmission coefficients for the probe waves propagating in the direction of pump and in the opposite direction become different, as do the corresponding reflection coefficients. Using Eq. (10) we calculate $\delta T=\left|t_{\uparrow \uparrow}\right|^{2}-\left|t_{\uparrow \downarrow}\right|^{2}$ and $\delta R=\left|r_{\uparrow \uparrow}\right|^{2}-\left|r_{\uparrow \downarrow}\right|^{2}$ :

$\delta T(\omega)=\frac{8 \omega J}{m v^{2}} \operatorname{Im} r\left(\omega_{0}\right) \operatorname{Re} \frac{\left[1+r^{*}(\omega)\right] r(\omega)}{\left(\omega-\omega_{0}+i \gamma\right)^{2}-\Omega_{r}^{2}}$,
$\delta R(\omega)=-\frac{8 \omega J}{m v^{2}} \operatorname{Re} r\left(\omega_{0}\right) \operatorname{Im} \frac{|r(\omega)|^{2}}{\left(\omega-\omega_{0}+i \gamma\right)^{2}-\Omega_{r}^{2}}$.

Figures 7(b) and 7(c) show the dependence of the nonreciprocal transmission $\delta T$ and reflection $\delta R$ on the frequencies of the pump and probe light for the case of a particle with resonant polarizability. The nonreciprocity arises when the detuning between pump and probe matches the mechanical frequency, $\omega-\omega_{0}= \pm \Omega_{r}$, enabling excitation of vibrations. Note that the sign of $\delta T$ is inverted in the vicinity of material resonance frequency $\omega_{x}$, opening a way for tunable optomechanical nonreciprocity.

The emergence of the pump-induced scattering nonreciprocity can be understood as a result of an effective synthetic magnetic field, induced by the pump wave and acting upon the probe [39-41]. For multiple scatterers in a one-dimensional or two-dimensional array with properly engineered phase difference, this magnetic field can lead to the formation of the topological edge states of light and sound $[42,43]$. Such effects are out of the scope of the present study, where we focus on the chiral interaction for one scatterer only. 
The optical pump also modifies the properties of the mechanical motion [44]. The effect is described by the selfenergy correction

$$
\begin{aligned}
\Sigma(\Omega)= & \frac{i J}{2 m v^{2} \Omega_{r}}\left[\left(\omega_{0}+\Omega\right) r\left(\omega_{0}+\Omega\right)\right. \\
& -\left(\omega_{0}-\Omega\right) r^{*}\left(\omega_{0}-\Omega\right)-2 \Omega\left|r\left(\omega_{0}\right)\right|^{2} \\
& \left.-2 i \omega_{0} \operatorname{Im} r\left(\omega_{0}\right)\right]
\end{aligned}
$$

derived in Appendix D. The real and imaginary parts of self-energy represent the correction to the mechanical eigenfrequency and damping, $\Sigma\left(\Omega_{r}\right)=\delta \Omega_{r}-i \delta \gamma$. If the mechanical frequency $\Omega_{r}$ is smaller than the width of the reflection coefficient resonances, the corrections describing optomechanical spring and heating or cooling effects are given by

$$
\begin{gathered}
\delta \Omega_{r}=-\frac{J \Omega_{r}}{2 m v^{2}} \operatorname{Im} \frac{d^{2}}{d \omega^{2}}(\omega r), \\
\delta \gamma=\frac{J}{m v^{2}}\left[|r|^{2}-\operatorname{Re} \frac{d}{d \omega}(\omega r)\right] .
\end{gathered}
$$

In the absence of optical losses the optical theorem yields $\operatorname{Re} r=-|r|^{2}$, so Eq. (14) is simplified to $\delta \gamma=$ $\left(J / m v^{2}\right)\left[2|r|^{2}+\omega d|r|^{2} / d \omega\right]$. Therefore, optomechanical heating or cooling effects are realized when the reflection coefficient decays or grows with the frequency, respectively.

\section{CONCLUSION}

To conclude, we discuss the possibilities for experimental realization of the optomechanical Kerker effect. The proof-of-principle observation of the suppression of the forward inelastic scattering in the nonresonant regime, Fig. 3(b), seems to be relatively straightforward for an arbitrary subwavelength particle. The only requirements are to operate in the far-field regime and to avoid the internal deformations of the particle, so that it trembles as a whole. The true challenge is to realize a dynamical tunability between forward and backward scattering by exploiting the resonance of particle polarizability. This requires the width of the resonance $\Gamma$ to be comparable with

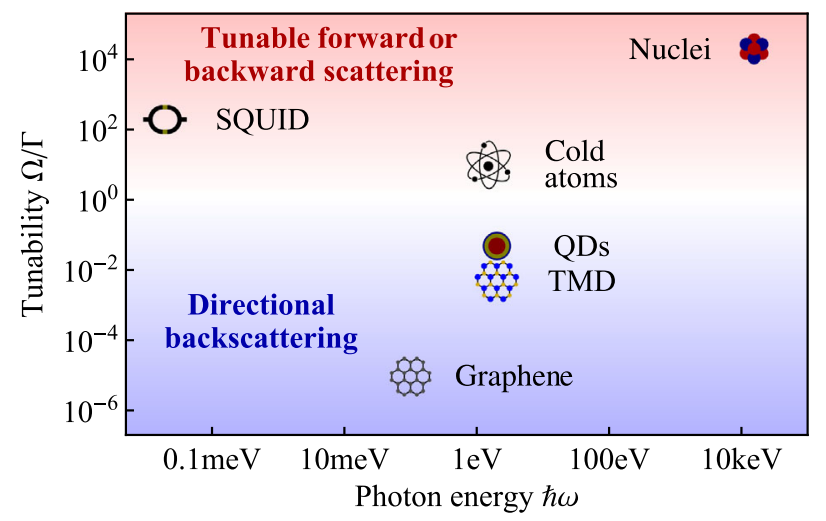

FIG. 8. Tunability of various resonant optomechanical systems operating in different spectral ranges. When $\Omega \ll \Gamma$ (blue area), the directional scattering can occur only in the backward direction. When $\Omega \gg \Gamma$ (red area), one can realize both directional forward and backward scattering by a proper tuning of the incident light frequency and trembling frequency. Numerical parameters and references are given in Table I.

the frequency of vibrations $\Omega$, i.e., narrow resonances and high vibration frequencies. Table I presents an overview of various potential systems in different ranges of the electromagnetic spectrum and Fig. 8 visualizes their tunability $\Omega / \Gamma$. Apparently, the highest degree of tunability can be attained by exploiting the Mössbauer resonances in the nuclei of crystals for $\gamma$ rays [45]. Namely, the linewidth can be as narrow as $0.5 \mathrm{MHz}$ [46], while the gigahertz-range hypersound waves in metals are available [47]. Further opportunities are provided by organizing planar cavities for synchrotron $\gamma$ radiation [48]. The opposite side of the electromagnetic spectrum, with sub-0.1-meV rather than $10-\mathrm{keV}$ photon energies, is represented by the superconducting qubits [49]. They feature a high quality factor resulting in potentially high tunability when being coupled to the ultrasound generator [50]. An apparent drawback of such a setup is a relatively weak scattering efficiency due to the vibration amplitude being relatively small as compared to the electromagnetic wavelength; see Table I. The terahertz and optical frequency ranges are accessible by membranes made of graphene [51] and transition-metal dichalcogenide monolayers (TMDs) [52], respectively. These platforms feature reasonable coupling strength but have limited tunability because of the relatively low

TABLE I. Parameters of various resonant optomechanical systems.

\begin{tabular}{lcccccc}
\hline \hline System & $\hbar \omega_{x}$ & $\Gamma /(2 \pi)$ & $\Omega /(2 \pi)$ & $u$ & Tunability $(\Omega / \Gamma)$ & Coupling $\left[\left(\omega_{x} / c\right) u\right]$ \\
\hline Plasmon in graphene [51,54,55] & $0.1-1 \mathrm{eV}$ & $10 \mathrm{THz}$ & $0.1 \mathrm{GHz}$ & $10 \mathrm{~nm}$ & $10^{-5}$ & $0.01-0.1$ \\
Exciton in TMD monolayers [52,55] & $2 \mathrm{eV}$ & $20 \mathrm{GHz}$ & $0.1 \mathrm{GHz}$ & $10 \mathrm{~nm}$ & $5 \times 10^{-3}$ & 0.1 \\
Exciton in colloidal QDs [50,56] & $2 \mathrm{eV}$ & $400 \mathrm{MHz}$ & $20 \mathrm{MHz}$ & $200 \mathrm{~nm}$ & $5 \times 10^{-2}$ & 2 \\
Cold atoms [53] & $1.5 \mathrm{eV}$ & $10 \mathrm{kHz}$ & $100 \mathrm{kHz}$ & $200 \mathrm{~nm}$ & 10 & 1.5 \\
Superconducting qubits [49,50] & $20 \mu \mathrm{eV}$ & $0.1 \mathrm{MHz}$ & $20 \mathrm{MHz}$ & $200 \mathrm{~nm}$ & 200 & $2 \times 10^{-5}$ \\
Resonance in nuclei [46,47] & $15 \mathrm{keV}$ & $0.5 \mathrm{MHz}$ & $10 \mathrm{GHz}$ & $10^{-3} \mathrm{~nm}$ & $2 \times 10^{4}$ & 0.1 \\
\hline \hline
\end{tabular}


frequency of the flexural vibrations $(\lesssim 0.1 \mathrm{GHz})$ as compared to the broad width of plasmonic or excitonic resonance. High tunability and strong optomechanical coupling efficiency for visible light can be realized by exploiting narrow resonances in cold atoms vibrating in an optical trap [53]. Alternatively, one could use excitonic resonances in artificial atoms, colloidal quantum dots (QDs)[50].

The rich consequences of the interplay of magnetic and electric response on the electromagnetic wave propagation have been known for at least 50 years, since the seminal work by Veselago on the media with negative permittivity and permeability [57,58]. Still, the mutual effect of electric and magnetic resonances is very far from being completely understood. For example, it has been realized only quite recently that the interference and coupling of electric and magnetic resonances underpin bianisotropic photonic topological insulators $[59,60]$, where the light backscattering on disorder is suppressed. We demonstrate that the proposed optomechanical Kerker and spin-Hall effects with trembling-induced magnetic response open a pathway to engineer chiral optomechanical coupling at the nanoscale, expanding chiral quantum optics [32,33] to the optomechanical domain. Our results can be instructive for the design of nonreciprocal topological circuits $[61,62]$, where the disorder-robust propagation of light and sound is ensured by the time modulation of optical and mechanical properties $[41,63,64]$.

\section{ACKNOWLEDGMENTS}

The authors acknowledge fruitful discussions with I. D. Avdeev, A. A. Bogdanov, N. A. Gippius, A. Krasnok, C. R. Simovski, A. Slobozhanyuk, and S. G. Tikhodeev. A. N. P. has been partially supported by the Australian Research Council. The authors also acknowledge support by the Russian President Grant No. MD-5791.2018, the RFBR Projects No. 18-32-00486, No. 17-52-16020, and No. 1702-00383 the Foundation for the Advancement of Theoretical Physics and Mathematics "BASIS", and Presidium of RAS (Program 32, Nanomaterials).

\section{APPENDIX A: POLARIZATION OF TREMBLING MEDIA}

We consider light with frequency $\omega$ incident on a medium vibrating with frequency $\Omega$. The medium motion is described by the displacement vector $\boldsymbol{u}(\boldsymbol{r}, t)=$ $\boldsymbol{u}(\boldsymbol{r}) e^{-i \Omega t}+$ c.c. We suppose that the vibration amplitude is small and focus on the linear-in- $\boldsymbol{u}$ effect only, i.e., the appearance of polarization at anti-Stokes- and Stokesshifted frequencies $\omega \pm \Omega$.

Consider the unitary volume of a medium that in the absence of vibration has coordinate $\boldsymbol{r}$. Its polarization at time $t$ is determined by the electric field $\tilde{\boldsymbol{E}}\left(\boldsymbol{r}, t^{\prime}\right)$ that has acted on it in all previous moments of time $t^{\prime}<t$,

$$
\tilde{\boldsymbol{P}}(\boldsymbol{r}, t)=\int_{-\infty}^{t} d t^{\prime} \chi\left(\boldsymbol{r}, t-t^{\prime}\right) \tilde{\boldsymbol{E}}\left(\boldsymbol{r}, t^{\prime}\right),
$$

where $\chi(\boldsymbol{r}, \tau)$ is the dielectric susceptibility function. Here, we do not account for the possible change of susceptibility under medium deformation, because such a photoelastic effect requires separate microscopic calculation. While photoelasticity may give dominant contribution to optomechanical coupling in resonant structures [65-68], it does not play any role in the effects we consider, where the objects move as a whole and deformation is absent. The electric field $\tilde{\boldsymbol{E}}\left(\boldsymbol{r}, t^{\prime}\right)$ in Eq. (A1) should be calculated in the reference frame that moves and rotates together with the considered medium volume. Keeping linear-in- $\boldsymbol{u}$ terms only, we obtain

$$
\tilde{\boldsymbol{E}}=\boldsymbol{E}+\left(\boldsymbol{u} \cdot \frac{\partial}{\partial \boldsymbol{r}}\right) \boldsymbol{E}-\frac{\operatorname{rot} \boldsymbol{u}}{2} \times \boldsymbol{E}+\frac{1}{c} \frac{\partial \boldsymbol{u}}{\partial t} \times \boldsymbol{B},
$$

where $\boldsymbol{E}$ and $\boldsymbol{B}$ are the electric and magnetic fields in the reference frame at rest, and all quantities are evaluated at the moment $t^{\prime}$. The second term in the right-hand side of Eq. (A2) stems from the fact that the electric field should be evaluated at the point $\boldsymbol{r}+\boldsymbol{u}\left(\boldsymbol{r}, t^{\prime}\right)$, the third term accounts for the medium rotation, and the last term comes from the Lorentz transform.

Equation (A1) gives the polarization of the unitary volume of an undeformed medium in the reference frame that moves and rotates with the medium. In the reference fame at rest, the polarization density reads

$$
\boldsymbol{P}=\tilde{\boldsymbol{P}}-\left(\boldsymbol{u} \cdot \frac{\partial}{\partial \boldsymbol{r}}\right) \tilde{\boldsymbol{P}}+\frac{\operatorname{rot} \boldsymbol{u}}{2} \times \tilde{\boldsymbol{P}}-\tilde{\boldsymbol{P}} \operatorname{div} \boldsymbol{u}
$$

where the last term accounts for the difference between the deformed and undeformed unitary volumes. Additionally, the magnetization $\boldsymbol{M}=-\partial \boldsymbol{u} / \partial t \times \tilde{\boldsymbol{P}}$ appears in the frame at rest due to the Lorentz transform.

Finally, we combine Eqs. (A1)-(A3) and evaluate the current $\boldsymbol{j}=\partial \boldsymbol{P} / \partial t+\operatorname{rot} \boldsymbol{M}$ in the reference frame at rest. The relation between the current $\boldsymbol{j}$ at the anti-Stokes-shifted frequency $\omega^{\prime}=\omega+\Omega$ and the vector potential $\boldsymbol{A}$ of light at the initial frequency $\omega$ in $\boldsymbol{k}$ space assumes the form $\boldsymbol{j}_{\boldsymbol{k}^{\prime}}\left(\omega^{\prime}\right)=\sum_{\boldsymbol{k}} \delta \boldsymbol{\Pi}_{\boldsymbol{k}^{\prime}, \boldsymbol{k}} \boldsymbol{A}_{\boldsymbol{k}}(\omega)$, where we use the gauge with zero scalar potential,

$$
\begin{aligned}
\delta \boldsymbol{\Pi}_{\boldsymbol{k}^{\prime}, k}= & \omega^{\prime 2} \chi_{\boldsymbol{k}^{\prime}+\boldsymbol{q}-\boldsymbol{k}}\left(\omega^{\prime}\right) \boldsymbol{\Lambda}_{\boldsymbol{k}+\boldsymbol{q}, \boldsymbol{k}}\left(\omega^{\prime}, \omega\right) \\
& +\boldsymbol{\Lambda}_{q-\boldsymbol{k}^{\prime},-\boldsymbol{k}^{\prime}}^{\boldsymbol{T}}\left(-\omega,-\omega^{\prime}\right) \omega^{2} \chi_{\boldsymbol{k}^{\prime}+\boldsymbol{q}-\boldsymbol{k}}(\omega),
\end{aligned}
$$

$\chi_{\boldsymbol{q}}(\omega)=\iint \chi(\boldsymbol{r}, \tau) e^{i \omega \tau-i \boldsymbol{q} \cdot \boldsymbol{r}} d \tau d^{3} r$ and $\boldsymbol{u}_{\boldsymbol{q}}$ are the spatial Fourier transforms of $[\varepsilon(\boldsymbol{r}, \omega)-1] /(4 \pi)$ and $\boldsymbol{u}(\boldsymbol{r})$, respectively, superscript $T$ denotes matrix transposition, and 
(a)

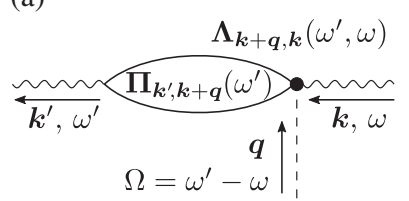

(c)

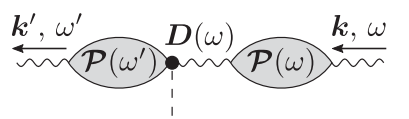

(b)

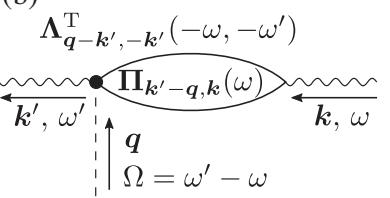

(d)

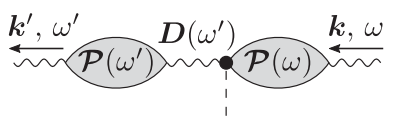

FIG. 9. (a),(b) Diagrammatic representation for the optomechanical interaction of light (wavy line), medium polarization (bubble), and mechanical vibration (dashed line). (c),(d) The contributions to the amplitude of light scattering by a trembling medium that together with contributions shown in Figs. 2(b) and 2(c) give the full amplitude of inelastic light scattering. Wavy line denotes photon and corresponds to the Green's function for vector potential $\boldsymbol{D}(\boldsymbol{k}, \omega)=-4 \pi\left(1-\boldsymbol{k} \otimes \boldsymbol{k} / \omega^{2}\right) /\left(\omega^{2}-\boldsymbol{k}^{2}\right)$, empty and filled bubbles correspond to the bare and dressed polarization operators of the medium at rest, $\boldsymbol{\Pi}$ and $\mathcal{P}=\boldsymbol{\Pi}(1-\boldsymbol{D} \boldsymbol{\Pi})^{-1}$, respectively, dashed line represents mechanical displacement $\boldsymbol{u}_{\boldsymbol{q}}$, solid dot is the optomechanical interaction $\boldsymbol{\Lambda}$ given by Eq. (A5).

$$
\begin{aligned}
\Lambda_{\boldsymbol{k}+\boldsymbol{q}, \boldsymbol{k}}\left(\omega^{\prime}, \omega\right)= & i\left(\boldsymbol{u}_{\boldsymbol{q}} \cdot \boldsymbol{k}\right)-i \frac{\omega^{\prime}-\omega}{\omega^{\prime}} \boldsymbol{k} \otimes \boldsymbol{u}_{\boldsymbol{q}} \\
& -i \frac{\omega}{\omega^{\prime}} \frac{\boldsymbol{u}_{\boldsymbol{q}} \otimes \boldsymbol{q}-\boldsymbol{q} \otimes \boldsymbol{u}_{\boldsymbol{q}}}{2}
\end{aligned}
$$

To calculate the polarization at the Stokes-shifted frequency $\omega-\Omega$, one should change in the above equations the sign of $\Omega$ and replace $\boldsymbol{u}_{\boldsymbol{q}}$ with $\boldsymbol{u}_{-\boldsymbol{q}}^{*}$. The quantity $\delta \boldsymbol{\Pi}$ is the correction to the polarization operator caused by the medium vibration. The two terms in the right-hand side of Eq. (A3) can be represented diagrammatically as shown in Figs. 9(a) and 9(b). The wavy line corresponds to a photon, the dashed line is a vibration, the bubble stands for polarization operator of medium at rest, $\boldsymbol{\Pi}_{\boldsymbol{k}^{\prime}, \boldsymbol{k}}(\omega)=$ $\omega^{2} \chi_{k^{\prime}-k}(\omega)$, and the solid dot represents optomechanical interaction $\boldsymbol{\Lambda}$.

\section{APPENDIX B: LIGHT SCATTERING ON TREMBLING OBJECTS}

Here we describe how the amplitude of inelastic light scattering on a trembling object of an arbitrary shape can be calculated. We use $c=1$ for simplicity. The full amplitude can be represented as a sum of four terms diagrammatically shown in Figs. 2(b), 2(c), 9(c), and 9(d). They correspond to the medium polarization, described by the dressed polarization operator $\mathcal{P}=\boldsymbol{\Pi}(1-\boldsymbol{D} \boldsymbol{\Pi})^{-1}$, accounted for before, after, or both before and after the optomechanical interaction.

Note that the photon Green's function $D_{k}(\omega)=-4 \pi(1-$ $\left.\boldsymbol{k} \otimes \boldsymbol{k} / \omega^{2}\right) /\left(\omega^{2}-\boldsymbol{k}^{2}\right)$ is $\boldsymbol{k}$-even while the optomechanical interaction $\boldsymbol{\Lambda}$ is $\boldsymbol{k}$-odd in the absence of the last term

describing medium rotation; see Eq. (A5). In the main text, we consider the small object characterized by the wavevector-independent dressed polarization operator $\mathcal{P}_{\boldsymbol{k}^{\prime}, \boldsymbol{k}}(\omega)=$ $\omega^{2} \boldsymbol{\alpha}(\omega)$. In such a case, the summation over the phonon wave vectors in the intermediate states of the diagrams Figs. 9(c) and 9(d) yields zero. The scattering amplitude is then given by the remaining diagrams Fig. 2(b) and 2(c).

In the general case, all the diagrams of Fig. 9 contribute to the total scattering matrix element that reads

$M_{\boldsymbol{k}^{\prime} \boldsymbol{k}}=\boldsymbol{A}_{\boldsymbol{k}^{\prime}}^{*} \cdot(1+\mathcal{P} \boldsymbol{D})\left(\boldsymbol{\Lambda}^{\boldsymbol{T}} \boldsymbol{\Pi}+\boldsymbol{\Pi} \mathbf{\Lambda}\right)(1+\boldsymbol{D} \mathcal{P}) \boldsymbol{A}_{\boldsymbol{k}}$,

where $\boldsymbol{A}_{\boldsymbol{k}}=\sqrt{2 \pi / \omega} \boldsymbol{e}_{0} e^{i \boldsymbol{k} \cdot \boldsymbol{r}}$ and $\boldsymbol{A}_{\boldsymbol{k}^{\prime}}=\sqrt{2 \pi / \omega^{\prime}} \boldsymbol{e}^{\prime} e^{i \boldsymbol{k}^{\prime} \cdot \boldsymbol{r}}$ are the vector potentials of the incident and scattered photons, and $\boldsymbol{e}_{0}$ and $\boldsymbol{e}^{\prime}$ are their polarizations. Introducing the field distributions of incident and scattered photons in the form $\mathcal{A}_{0}(\boldsymbol{r})=(1+\boldsymbol{D} \mathcal{P}) \boldsymbol{A}_{\boldsymbol{k}}$ and $\mathcal{A}^{\prime *}(\boldsymbol{r})=\left(1+\boldsymbol{D} \mathcal{P}^{T}\right) \boldsymbol{A}_{\boldsymbol{k}^{\prime}}^{*}$, we finally obtain the scattering amplitude per solid angle $d o$ :

$$
\begin{aligned}
\frac{d S_{\boldsymbol{k}^{\prime} \boldsymbol{k}}}{d o}= & \frac{i \omega^{\prime 2}}{(2 \pi)^{2}} \int\left[\boldsymbol{\Pi}^{\boldsymbol{T}}\left(\boldsymbol{r}, \omega^{\prime}\right) \mathcal{A}^{*}(\boldsymbol{r}) \cdot \boldsymbol{\Lambda}\left(\omega^{\prime}, \omega\right) \mathcal{A}_{0}(\boldsymbol{r})\right. \\
& \left.+\boldsymbol{\Lambda}\left(-\omega,-\omega^{\prime}\right) \mathcal{A}^{* *}(\boldsymbol{r}) \cdot \boldsymbol{\Pi}(\boldsymbol{r}, \omega) \mathcal{A}_{0}(\boldsymbol{r})\right] d \boldsymbol{r}
\end{aligned}
$$

Here, the operator $\boldsymbol{\Lambda}$ with the components

$$
\begin{aligned}
\Lambda_{\alpha \beta}\left(\omega^{\prime}, \omega\right)= & \left(\boldsymbol{u} \cdot \frac{\partial}{\partial \boldsymbol{r}}\right) \delta_{\alpha \beta}-\frac{\omega^{\prime}-\omega}{\omega^{\prime}} u_{\beta} \frac{\partial}{\partial r_{\alpha}} \\
& +\frac{1}{2} \frac{\omega}{\omega^{\prime}} \epsilon_{\alpha \beta \gamma}(\operatorname{rot} \boldsymbol{u})_{\gamma}
\end{aligned}
$$

is the optomechanical interaction operator Eq. (A5) in the coordinate representation, and the polarizability operator can be readily expressed via the dielectric permittivity as $\boldsymbol{\Pi}(\boldsymbol{r}, \omega)=\omega^{2}[\boldsymbol{\varepsilon}(\boldsymbol{r}, \omega)-1] / 4 \pi$. When the time-inversion symmetry holds, the dielectric permittivity tensor is symmetric, so $\boldsymbol{\Pi}^{\boldsymbol{T}}=\boldsymbol{\Pi}$. Then the distributions $\mathcal{A}_{0}(\boldsymbol{r})$ and $\mathcal{A}^{\prime *}(\boldsymbol{r})$ can be calculated as the fields induced in the system by the light incident with wave vectors $\boldsymbol{k}$ and $-\boldsymbol{k}^{\prime}$, respectively.

\section{APPENDIX C: LIGHT SCATTERING BY A VIBRATING RESONANT LAYER}

We derive here the amplitudes of light scattering on a vibrating resonant layer, Eq. (8). The layer is described by a dielectric susceptibility tensor with the components $\chi_{\alpha \beta}(\boldsymbol{r}, \omega)=\delta_{\alpha \beta} \delta(z) \chi(\omega)$ and $\chi_{z z}=\chi_{\alpha z}=\chi_{z \alpha}=0$. Here $\alpha, \beta=x, y$ are the in-plane coordinates and $z$ is the layer normal. Using the Green's function

$$
D_{\alpha \beta}(\boldsymbol{k}, z, \omega)=\frac{2 \pi i}{k_{z}}\left(\delta_{\alpha \beta}-\frac{k_{\alpha} k_{\beta}}{\omega^{2}}\right) e^{i k_{z}|z|},
$$

where $\boldsymbol{k}=\left(k_{x}, k_{y}\right)$ is the in-plane wave vector and $k_{z}=$ $\sqrt{\omega^{2}-\boldsymbol{k}^{2}}$, the dressed polarization operator of the layer $\mathcal{P}=\boldsymbol{\Pi}(1-\boldsymbol{D} \boldsymbol{\Pi})^{-1}$ can be evaluated. We find 


$$
\begin{aligned}
\mathcal{P}_{\alpha \beta}(\boldsymbol{k}, z, \omega)= & \frac{\omega^{2} \chi(\omega) \delta(z)}{1-2 \pi i \omega^{2} \chi(\omega) / k_{z}}\left(\delta_{\alpha \beta}-\frac{k_{\alpha} k_{\beta}}{\boldsymbol{k}^{2}}\right) \\
& +\frac{\omega^{2} \chi(\omega) \delta(z)}{1-2 \pi i k_{z} \chi(\omega)} \frac{k_{\alpha} k_{\beta}}{\boldsymbol{k}^{2}}
\end{aligned}
$$

and $\mathcal{P}_{z z}=\mathcal{P}_{\alpha z}=\mathcal{P}_{z \alpha}=0$. The amplitude of coherent light reflection from the layer is given by $r=\boldsymbol{e}^{* *} \cdot\left(2 \pi i \mathcal{P} / k_{z}\right) \boldsymbol{e}_{0}$. For $s$ - and $p$-polarized light we obtain

$$
\begin{gathered}
r_{s}(\boldsymbol{k}, \omega)=\frac{2 \pi i \omega^{2} \chi(\omega) / k_{z}}{1-2 \pi i \omega^{2} \chi(\omega) / k_{z}}, \\
r_{p}(\boldsymbol{k}, \omega)=\frac{2 \pi i k_{z} \chi(\omega)}{1-2 \pi i k_{z} \chi(\omega)}
\end{gathered}
$$

To calculate the amplitudes of light scattered by a layer vibration, we use the approach described in Appendix B. First, we calculate the vector potential distribution induced by a photon at the frequency $\omega$ incident from $z \rightarrow-\infty$ with the in-plane wave vector $\boldsymbol{k}$ and the polarization $\boldsymbol{e}$,

$$
\begin{aligned}
\mathcal{A}_{\omega, \boldsymbol{k}}(\boldsymbol{r})= & \sqrt{\frac{2 \pi}{\omega}} e^{i \boldsymbol{k} \cdot \boldsymbol{r}}\left\{\boldsymbol{e} e^{i k_{z} z}+\left[r_{s}(\boldsymbol{k}, \omega) \boldsymbol{e}_{t}\right.\right. \\
& \left.\left.+r_{p}(\boldsymbol{k}, \omega) \boldsymbol{e}_{l}+\operatorname{sgn} z r_{p}(\boldsymbol{k}, \omega) \boldsymbol{e}_{z}\right] e^{i k_{z}|z|}\right\},
\end{aligned}
$$

where $\boldsymbol{e}=\boldsymbol{e}_{l}+\boldsymbol{e}_{t}+\boldsymbol{e}_{z}$ with $\boldsymbol{e}_{z} \| z$ being the out-of-plane component of the light polarization vector $\boldsymbol{e}$, and $\boldsymbol{e}_{l} \| \boldsymbol{k}, \boldsymbol{e}_{t} \perp \boldsymbol{k}$ being its in-plane components. The layer polarization induced by the photon reads

$$
\begin{aligned}
\boldsymbol{\Pi}(\boldsymbol{r}, \omega) \mathcal{A}_{\omega, \boldsymbol{k}}(\boldsymbol{r})= & -\frac{i k_{z} \delta(z)}{\sqrt{2 \pi \omega}} e^{i \boldsymbol{k} \cdot \boldsymbol{r}} \\
& \times\left[r_{s}(\boldsymbol{k}, \omega) \boldsymbol{e}_{t}+\frac{\omega^{2}}{k_{z}^{2}} r_{p}(\boldsymbol{k}, \omega) \boldsymbol{e}_{l}\right] .
\end{aligned}
$$

Then we calculate $\boldsymbol{\Lambda}\left(\omega^{\prime}, \omega\right) \mathcal{A}_{\omega, \boldsymbol{k}}(\boldsymbol{r})$. Keeping in mind that according to Eq. (B2) the result is to be multiplied by $\boldsymbol{\Pi}(\boldsymbol{r}, \omega) \mathcal{A}_{\omega, k}(\boldsymbol{r})$, all we need are the in-plane components of $\Lambda\left(\omega^{\prime}, \omega\right) \mathcal{A}_{\omega, k}(\boldsymbol{r})$ at $z=0$. They read

$$
\begin{aligned}
\boldsymbol{\Lambda}\left(\omega^{\prime}, \omega\right) \mathcal{A}_{\omega, \boldsymbol{k}}= & i \sqrt{\frac{2 \pi}{\omega}}\left[k_{z}\left(\boldsymbol{e}_{t}+\boldsymbol{e}_{l}\right)+\frac{\Omega}{\omega^{\prime}} \frac{\boldsymbol{k}^{2}}{k_{z}} \boldsymbol{e}_{l}\right. \\
& \left.-\frac{\omega}{\omega^{\prime}} \frac{\boldsymbol{e}_{l} \cdot \boldsymbol{k}}{k_{z}} \boldsymbol{q}+(\cdots) \boldsymbol{e}_{z}\right] u_{z} e^{i(\boldsymbol{k}+\boldsymbol{q}) \cdot \boldsymbol{r}},
\end{aligned}
$$

where the dots replace the out-of-plane component. Note that when evaluating the action of the optomechanical interaction operator Eq. (B3), in the last term describing the layer rotation we used $\operatorname{rot} \boldsymbol{u}=2\left(\partial u_{z} / \partial y,-\partial u_{z} / \partial x, 0\right)$. The factor 2 arises from the contribution of $\partial u_{\alpha} / \partial z(\alpha=x, y)$ that are nonzero even though $u_{\alpha}=0$ at $z=0$.

Finally, we calculate the backward and forward photon scattering amplitudes:

$$
\begin{aligned}
R_{\rightarrow(\hookleftarrow)}= & i \frac{\omega^{\prime}}{k_{z}^{\prime}} u_{z} \int\left[\boldsymbol{\Pi} \mathcal{A}_{\omega^{\prime},-\boldsymbol{k}^{\prime}} \cdot \boldsymbol{\Lambda}\left(\omega^{\prime}, \omega\right) \mathcal{A}_{\omega, \boldsymbol{k}}\right. \\
& \left.\mp \boldsymbol{\Lambda}\left(-\omega,-\omega^{\prime}\right) \mathcal{A}_{\omega^{\prime},-\boldsymbol{k}} \cdot \boldsymbol{\Pi} \mathcal{A}_{\omega, \boldsymbol{k}}\right] d z .
\end{aligned}
$$

We make use of Eqs. (C6) and (C7) and take into account that for the photon incident from $z \rightarrow+\infty$, the in-plane components of $\Lambda \mathcal{A}$ have opposite signs, while $\Pi \mathcal{A}$ is the same. Finally, we obtain

$$
\begin{aligned}
& R_{\rightarrow(\hookleftarrow)} \\
& =i \sqrt{\frac{\omega^{\prime}}{\omega}} u_{z}\left\{\left[r_{s}^{\prime} \boldsymbol{e}_{t}^{\prime}+\frac{\omega^{\prime 2}}{k_{z}^{\prime 2}} r_{p}^{\prime} \boldsymbol{e}_{l}^{\prime}\right] \cdot\left[k_{z}\left(\boldsymbol{e}_{t}+\boldsymbol{e}_{l}\right)+\frac{\boldsymbol{e}_{l} \cdot \boldsymbol{k}}{k_{z}}\left(\boldsymbol{k}-\frac{\omega}{\omega^{\prime}} \boldsymbol{k}^{\prime}\right)\right]\right. \\
& \left.\quad \pm \frac{k_{z}}{k_{z}^{\prime}}\left[k_{z}^{\prime}\left(\boldsymbol{e}_{t}^{\prime}+\boldsymbol{e}_{l}^{\prime}\right)+\frac{\boldsymbol{e}_{l}^{\prime} \cdot \boldsymbol{k}^{\prime}}{k_{z}^{\prime}}\left(\boldsymbol{k}^{\prime}-\frac{\omega^{\prime}}{\omega} \boldsymbol{k}\right)\right] \cdot\left[r_{s} \boldsymbol{e}_{t}+\frac{\omega^{2}}{k_{z}^{2}} r_{p} \boldsymbol{e}_{l}\right]\right\},
\end{aligned}
$$

where the quantities without (with) a prime refer to the initial (scattered) photon. Multiplying the result by the factor $\sqrt{\omega^{\prime} / \omega}$ to relate electric fields rather than the photon amplitudes and considering $\boldsymbol{e}$ and $\boldsymbol{e}^{\prime}$ that represent $s$ and $p$ polarizations, we recover Eq. (8) of the main text.

\section{APPENDIX D: OPTOMECHANICAL SELF-ENERGY CORRECTION}

The optical excitation of the optomechanical system leads to modification of the mechanical motion properties. The modification can be described by the self-energy correction to particle vibration frequency $\Omega_{r}$ due to the processes where the vibration quantum is converted to an anti-Stokes or Stokes photon and then converted back; see upper four diagrams in Fig. 10. For the sake of simplicity, we disregard here the light polarization and consider the
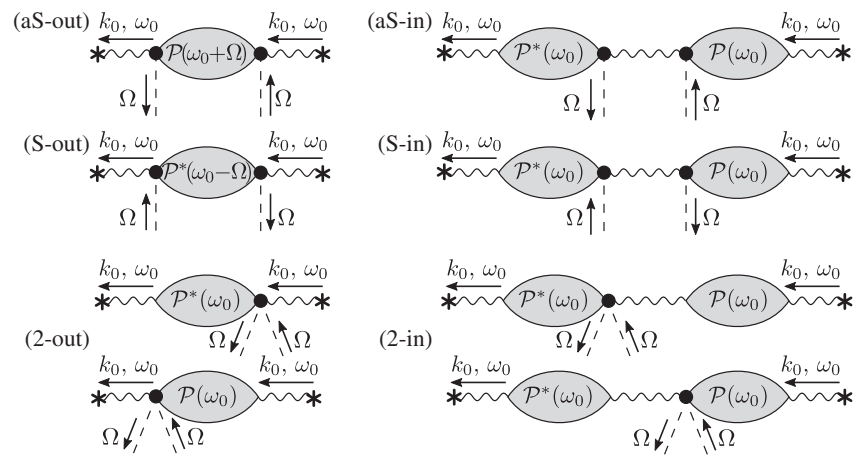

FIG. 10. Diagrammatic representation for the optomechanical correction to the mechanical motion self-energy. Star stands for the optical pump. In the four upper diagrams, the solid dot denotes the first-order optomechanical interaction, Eq. (B3). In the four lower diagrams, the solid dot represents the second-order optomechanical interaction described by $\Lambda^{(2)}=-|u|^{2} k^{2}$, where $k$ is the wave vector of the involved photon, and $u$ is the displacement. 
scalar problem. The optomechanical interaction vortex is then limited to the first term of Eq. (B3). The contributions to self-energy assume the form

$$
\begin{aligned}
\Sigma_{\mathrm{aS}-\mathrm{out}} & =-\omega_{0}^{2}|u|^{2} \mathcal{P}\left(\omega_{0}+\Omega\right)\left|A_{0}\right|^{2}, \\
\Sigma_{\mathrm{S}-\mathrm{out}} & =-\omega_{0}^{2}|u|^{2} \mathcal{P}^{*}\left(\omega_{0}-\Omega\right)\left|A_{0}\right|^{2}, \\
\Sigma_{\mathrm{aS}-\text { in }} & =|u|^{2}\left|\mathcal{P}\left(\omega_{0}\right)\right|^{2}\left|A_{0}\right|^{2} \int \frac{4 \pi k^{2}}{\left(\omega_{0}+\Omega+i 0\right)^{2}-k^{2}} \frac{d k}{2 \pi}, \\
\Sigma_{\mathrm{S} \text {-in }} & =|u|^{2}\left|\mathcal{P}\left(\omega_{0}\right)\right|^{2}\left|A_{0}\right|^{2} \int \frac{4 \pi k^{2}}{(\omega-\Omega-i 0)^{2}-k^{2}} \frac{d k}{2 \pi} .
\end{aligned}
$$

Additionally, the second-order optomechanical interaction, that corresponds to simultaneous vibration absorption and emission, should be taken into account. The processes featuring such interaction are depicted as the four lower diagrams in Fig. 10 and yield the self-energy corrections

$$
\begin{aligned}
\Sigma_{2 \text {-out }} & =-2|u|^{2}\left|\mathcal{P}\left(\omega_{0}\right)\right|^{2}\left|A_{0}\right|^{2} \operatorname{Re} \int \frac{4 \pi k^{2}}{\left(\omega_{0}+i 0\right)^{2}-k^{2}} \frac{d k}{2 \pi}, \\
\Sigma_{2 \text {-in }} & =2 \omega_{0}^{2}\left|u A_{0}\right|^{2} \operatorname{Re} \mathcal{P}\left(\omega_{0}\right) .
\end{aligned}
$$

We sum up all the above contributions, express the result via the reflection coefficient $r(\omega)=2 \pi i \mathcal{P}(\omega) / \omega$, take into account the vibration quanta normalization $|u|^{2}=$ $1 /\left(2 m \Omega_{r}\right)$ and obtain Eq. (12) of the main text.

[1] M. I. Mishchenko, L. D. Travis, and A. A. Lacis, Scattering, Absorption, and Emission of Light by Small Particles (Cambridge University Press, Cambridge, England, 2002).

[2] R. C. C. Leite, R. S. Moore, S. P. S. Porto, and J. E. Ripper, Angular Dependence of the Rayleigh Scattering from LowTurbidity Molecular Liquids, Phys. Rev. Lett. 14, 7 (1965).

[3] T. C. Damen, R. C. C. Leite, and S. P. S. Porto, Angular Dependence of the Raman Scattering from Benzene Excited by the He-Ne cw Laser, Phys. Rev. Lett. 14, 9 (1965).

[4] C. V. Raman, Molecular Diffraction of Light (Calcutta University Press, Calcutta, 1922).

[5] C. V. Raman, The Doppler Effect in the Molecular Scattering of Radiation, Nature (London) 103, 165 (1919).

[6] T. Hänsch and A. Schawlow, Cooling of Gases by Laser Radiation, Opt. Commun. 13, 68 (1975).

[7] A. H. Compton, A Quantum Theory of the Scattering of X-Rays by Light Elements, Phys. Rev. 21, 483 (1923).

[8] M. Kerker, D.-S. Wang, and C. L. Giles, Electromagnetic Scattering by Magnetic Spheres, J. Opt. Soc. Am. 73, 765 (1983).

[9] R. V. Mehta, R. Patel, R. Desai, R. V. Upadhyay, and K. Parekh, Experimental Evidence of Zero Forward Scattering by Magnetic Spheres, Phys. Rev. Lett. 96, 127402 (2006).

[10] J. Geffrin, B. García-Cámara, R. Gómez-Medina, P. Albella, L. Froufe-Pérez, C. Eyraud, A. Litman, R. Vaillon, F. González, M. Nieto-Vesperinas, J. Sáenz, and F. Moreno,
Magnetic and Electric Coherence in Forward-and BackScattered Electromagnetic Waves by a Single Dielectric Subwavelength Sphere, Nat. Commun. 3, 1171 (2012).

[11] W. Liu and Y.S. Kivshar, Generalized Kerker effects in Nanophotonics and Meta-Optics, Opt. Express 26, 13085 (2018).

[12] L. Landau and E. Lifshitz, Electrodynamics of Continuous Media (Pergamon, New York, 1974).

[13] A. I. Kuznetsov, A. E. Miroshnichenko, Y. H. Fu, J. Zhang, and B. Luk'yanchuk, Magnetic Light, Sci. Rep. 2, 492 (2012).

[14] A. I. Kuznetsov, A. E. Miroshnichenko, M. L. Brongersma, Y.S. Kivshar, and B. Luk'yanchuk, Optically Resonant Dielectric Nanostructures, Science 354, aag2472 (2016).

[15] M. F. Limonov, M. V. Rybin, A. N. Poddubny, and Y. S. Kivshar, Fano Resonances in Photonics, Nat. Photonics 11, 543 (2017).

[16] I. Staude, A. E. Miroshnichenko, M. Decker, N. T. Fofang, S. Liu, E. Gonzales, J. Dominguez, T. S. Luk, D. N. Neshev, I. Brener, and Y. Kivshar, Tailoring Directional Scattering through Magnetic and Electric Resonances in Subwavelength Silicon Nanodisks, ACS Nano 7, 7824 (2013).

[17] F. Ding, A. Pors, and S. I. Bozhevolnyi, Gradient Metasurfaces: A Review of Fundamentals and Applications, Rep. Prog. Phys. 81, 026401 (2018).

[18] J. Courtial, D. A. Robertson, K. Dholakia, L. Allen, and M. J. Padgett, Rotational Frequency Shift of a Light Beam, Phys. Rev. Lett. 81, 4828 (1998).

[19] S. V. Bulanov, T. Z. Esirkepov, M. Kando, A. S. Pirozhkov, and N. N. Rosanov, Relativistic Mirrors in PlasmasNovel Results and Perspectives, Usp. Fiz. Nauk 183, 449 (2013) [Phys. Usp. 56, 429 (2013)].

[20] V. Kozlov, D. Filonov, Y. Yankelevich, and P. Ginzburg, Micro-Doppler Frequency Comb Generation by Rotating Wire Scatterers, J. Quant. Spectrosc. Radiat. Transfer 190, 7 (2017).

[21] L. Novotny and B. Hecht, Principles of Nano-Optics (Cambridge University Press, New York, 2006).

[22] A. E. Krasnok, I. S. Maksymov, A. I. Denisyuk, P. A. Belov, A. E. Miroshnichenko, C. R. Simovski, and Y. S. Kivshar, Optical Nanoantennas, Phys. Usp., 56, 539 (2013).

[23] R. Harrington, On the Gain and Beamwidth of Directional Antennas, IRE Trans. Antennas Propag. 6, 219 (1958).

[24] R. Harrington, Effect of Antenna Size on Gain, Bandwidth, and Efficiency, J. Res. Natl. Bur. Stand., Sect. D 64, 1 (1960).

[25] A. Kavokin, G. Malpuech, and M. Glazov, Optical Spin Hall Effect, Phys. Rev. Lett. 95, 136601 (2005).

[26] C. Leyder, M. Romanelli, J. P. Karr, E. Giacobino, T. C. H. Liew, M. M. Glazov, A. V. Kavokin, G. Malpuech, and A. Bramati, Observation of the Optical Spin Hall Effect, Nat. Phys. 3, 628 (2007).

[27] K. Bliokh and Y. Bliokh, Topological Spin Transport of Photons: The Optical Magnus Effect and Berry Phase, Phys. Lett. A 333, 181 (2004).

[28] K. Y. Bliokh, F. J. Rodríguez-Fortuńo, F. Nori, and A. V. Zayats, Spin-Orbit Interactions of Light, Nat. Photonics 9 , 796 (2015).

[29] M. Dyakonov, Spin Physics in Semiconductors (SpringerVerlag, Berlin, 2008). 
[30] C. Pfeiffer and A. Grbic, Metamaterial Huygens' Surfaces: Tailoring Wave Fronts with Reflectionless Sheets, Phys. Rev. Lett. 110, 197401 (2013).

[31] E. L. Ivchenko, Optical Spectroscopy of Semiconductor Nanostructures (Alpha Science International, Harrow, England, 2005).

[32] P. Lodahl, S. Mahmoodian, S. Stobbe, A. Rauschenbeutel, P. Schneeweiss, J. Volz, H. Pichler, and P. Zoller, Chiral Quantum Optics, Nature (London) 541, 473 (2017).

[33] F. Spitzer, A. N. Poddubny, I. A. Akimov, V. F. Sapega, L. Klompmaker, L. E. Kreilkamp, L. V. Litvin, R. Jede, G. Karczewski, M. Wiater, T. Wojtowicz, D. R. Yakovlev, and M. Bayer, Routing the Emission of a Near-Surface Light Source by a Magnetic Field, Nat. Phys. 14, 1043 (2018).

[34] M. Hafezi and P. Rabl, Optomechanically Induced NonReciprocity in Microring Resonators, Opt. Express 20, 7672 (2012).

[35] S. Kim, X. Xu, J. M. Taylor, and G. Bahl, Dynamically Induced Robust Phonon Transport and Chiral Cooling in an Optomechanical System, Nat. Commun. 8, 205 (2017).

[36] J. Kim, M. C. Kuzyk, K. Han, H. Wang, and G. Bahl, NonReciprocal Brillouin Scattering Induced Transparency, Nat. Phys. 11, 275 (2015).

[37] F. Ruesink, M.-A. Miri, A. Alù, and E. Verhagen, Nonreciprocity and Magnetic-Free Isolation Based on Optomechanical Interactions, Nat. Commun. 7, 13662 (2016).

[38] Z. Shen, Y.-L. Zhang, Y. Chen, C.-L. Zou, Y.-F. Xiao, X.-B. Zou, F.-W. Sun, G.-C. Guo, and C.-H. Dong, Experimental Realization of Optomechanically Induced Non-Reciprocity, Nat. Photonics 10, 657 (2016).

[39] M. Schmidt, S. Kessler, V. Peano, O. Painter, and F. Marquardt, Optomechanical Creation of Magnetic Fields for Photons on a Lattice, Optica 2, 635 (2015).

[40] A. Celi, P. Massignan, J. Ruseckas, N. Goldman, I. B. Spielman, G. Juzeliūnas, and M. Lewenstein, Synthetic Gauge Fields in Synthetic Dimensions, Phys. Rev. Lett. 112, 043001 (2014).

[41] A. V. Poshakinskiy and A. N. Poddubny, Phonoritonic Crystals with a Synthetic Magnetic Field for an Acoustic Diode, Phys. Rev. Lett. 118, 156801 (2017).

[42] V. Peano, C. Brendel, M. Schmidt, and F. Marquardt, Topological Phases of Sound and Light, Phys. Rev. X 5, 031011 (2015).

[43] C. Brendel, V. Peano, O. J. Painter, and F. Marquardt, Pseudomagnetic Fields for Sound at the Nanoscale, Proc. Natl. Acad. Sci. U.S.A. 114, E3390 (2017).

[44] M. Aspelmeyer, T. J. Kippenberg, and F. Marquardt, Cavity Optomechanics, Rev. Mod. Phys. 86, 1391 (2014).

[45] R. Röhlsberger, Nuclear Resonant Scattering of Synchrotron Radiation from Thin Films, Hyperfine Interact. 123, 455 (1999).

[46] G. V. Smirnov, N. A. Semioshkina, V. V. Sklyarevskiŭ, S. Kadechkova, and B. Shestak, Energy Spectrum of $\gamma$ Rays Passing Anomalously through Resonantly Absorbing Fe ${ }^{57}$ Crystals, Zh. Eksp. Teor. Fiz. 72, 340 (1977) [JETP 45, 180 (1977)].

[47] G. Dewar, Electromagnetic Generation of Gigahertz Sound in Ferromagnetic Metals, Phys. Rev. B 36, 7805 (1987).
[48] R. Röhlsberger, K. Schlage, B. Sahoo, S. Couet, and R. Rüffer, Collective Lamb Shift in Single-Photon Superradiance, Science 328, 1248 (2010).

[49] H. Paik, D. I. Schuster, L. S. Bishop, G. Kirchmair, G. Catelani, A. P. Sears, B. R. Johnson, M. J. Reagor, L. Frunzio, L. I. Glazman, S. M. Girvin, M. H. Devoret, and R. J. Schoelkopf, Observation of High Coherence in Josephson Junction Qubits Measured in a Three-Dimensional Circuit QED Architecture, Phys. Rev. Lett. 107, 240501 (2011).

[50] S. Saffar and A. Abdullah, Simple Method for Measuring Vibration Amplitude of High Power Airborne Ultrasonic Transducer: Using Thermo-Couple, Ultrasonics 54, 821 (2014).

[51] C. Chen, S. Rosenblatt, K. I. Bolotin, W. Kalb, P. Kim, I. Kymissis, H. L. Stormer, T. F. Heinz, and J. Hone, Performance of Monolayer Graphene Nanomechanical Resonators with Electrical Readout, Nat. Nanotechnol. 4, 861 (2009).

[52] C. Samanta, P. R. Y. Gangavarapu, and A. K. Naik, Nonlinear Mode Coupling and Internal Resonances in $\mathrm{MoS}_{2}$ Nanoelectromechanical System, Appl. Phys. Lett. 107, 173110 (2015).

[53] P. S. Jessen, C. Gerz, P. D. Lett, W. D. Phillips, S. L. Rolston, R. J. C. Spreeuw, and C. I. Westbrook, Observation of Quantized Motion of Rb Atoms in an Optical Field, Phys. Rev. Lett. 69, 49 (1992).

[54] H. Yan, T. Low, W. Zhu, Y. Wu, M. Freitag, X. Li, F. Guinea, P. Avouris, and F. Xia, Damping Pathways of Mid-Infrared Plasmons in Graphene Nanostructures, Nat. Photonics 7, 394 (2013).

[55] D. Davidovikj, F. Alijani, S. J. Cartamil-Bueno, H. S. J. van der Zant, M. Amabili, and P. G. Steeneken, Nonlinear Dynamic Characterization of Two-Dimensional Materials, Nat. Commun. 8, 1253 (2017).

[56] M. J. Fernée, P. Tamarat, and B. Lounis, Spectroscopy of Single Nanocrystals, Chem. Soc. Rev. 43, 1311 (2014).

[57] V. G. Veselago, The Electrodynamics of Substances with Simultaneously Negative Values of $\epsilon$ and $\mu$, Usp. Fiz. Nauk 92, 517 (1967) [Sov. Phys. Usp. 10, 509 (1968)].

[58] V. G. Veselago and E. E. Narimanov, The Left Hand of Brightness: Past, Present and Future of Negative Index Materials, Nat. Mater. 5, 759 (2006).

[59] A. B. Khanikaev, S. Hossein Mousavi, W.-K. Tse, M. Kargarian, A.H. MacDonald, and G. Shvets, Photonic Topological Insulators, Nat. Mater. 12, 233 (2013).

[60] A. Slobozhanyuk, S. H. Mousavi, X. Ni, D. Smirnova, Y. S. Kivshar, and A.B. Khanikaev, Three-Dimensional AllDielectric Photonic Topological Insulator, Nat. Photonics 11, 130 (2017).

[61] L. Lu, J. D. Joannopoulos, and M. Soljačić, Topological States in Photonic Systems, Nat. Phys. 12, 626 (2016).

[62] T. Ozawa, H. M. Price, A. Amo, N. Goldman, M. Hafezi, L. Lu, M. Rechtsman, D. Schuster, J. Simon, O. Zilberberg, and I. Carusotto, Topological Photonics, arXiv:1802.04173 [Rev. Mod. Phys. (to be published)].

[63] D. L. Sounas and A. Alù, Non-Reciprocal Photonics Based on Time Modulation, Nat. Photonics 11, 774 (2017). 
[64] D. B. Sohn, S. Kim, and G. Bahl, Time-Reversal Symmetry Breaking with Acoustic Pumping of Nanophotonic Circuits, Nat. Photonics 12, 91 (2018).

[65] T. Berstermann, A. V. Scherbakov, A. V. Akimov, D. R. Yakovlev, N. A. Gippius, B. A. Glavin, I. Sagnes, J. Bloch, and M. Bayer, Terahertz Polariton Sidebands Generated by Ultrafast Strain Pulses in an Optical Semiconductor Microcavity, Phys. Rev. B 80, 075301 (2009).

[66] A. Fainstein, N. D. Lanzillotti-Kimura, B. Jusserand, and B. Perrin, Strong Optical-Mechanical Coupling in a Vertical
GaAs/AlAs Microcavity for Subterahertz. Phonons and Near-Infrared Light, Phys. Rev. Lett. 110, 037403 (2013).

[67] B. Jusserand, A. N. Poddubny, A. V. Poshakinskiy, A. Fainstein, and A. Lemaitre, Polariton Resonances for Ultrastrong Coupling Cavity Optomechanics in GaAs/ AlAs Multiple Quantum Wells, Phys. Rev. Lett. 115, 267402 (2015).

[68] A. V. Poshakinskiy, A. N. Poddubny, and A. Fainstein, Multiple Quantum Wells for $\mathcal{P} \mathcal{T}$-Symmetric Phononic Crystals, Phys. Rev. Lett. 117, 224302 (2016). 